\title{
Optimizing Fuel Treatments to Reduce Wildland Fire Risk
}

\author{
Woodam Chung
}

Published online: 25 January 2015

(C) Springer International Publishing AG 2015

\begin{abstract}
Fuel treatments have been widely used as an effective fire management tool to mitigate catastrophic wildland fire risk in forested landscapes. Fire research efforts of the last two decades have significantly advanced fire behavior modeling and fuel treatment effects analysis, but integrated fuel treatment planning and optimization models have yet to be extensively developed and used, mainly due to the complexity of the planning problem. This paper describes the problem complexity in terms of essential considerations when deciding where, when, and how to perform fuel treatments. Previous studies published in mainstream peer-reviewed journals are summarized and identified by their unique contributions, assumptions, and simplifications. Only a handful studies assessed fuel treatment effects in spatial and temporal contexts and incorporated them into the optimization framework. Most of these existing studies introduced optimization approaches as proof of concept with limited applications. It is hoped that future studies will build on these previous efforts and develop more efficient and integrated optimization approaches that can address multiple concerns simultaneously while producing effective fuel treatment plans for field implementation.
\end{abstract}

Keywords Fire management - Fire modeling - Mathematical programming $\cdot$ Heuristics $\cdot$ Large-scale optimization

This article is part of the Topical Collection on Integrating forestry in land use planning

W. Chung $(\bowtie)$

Department of Forest Engineering, Resources and Management, College of Forestry, Oregon State University, Corvallis, OR 97331, USA

e-mail: woodam.chung@oregonstate.edu

\section{Introduction}

Wildland fires are still viewed as a threat to society and ecosystems. Many efforts have been made to suppress and exclude fire from forest land management. For example, in the USA, the "10 Acre Policy" and "10 A.M. Policy," developed in the 1920s and 1930s by the US Forest Service, mandated all wildfires should be suppressed before they reached $10 \mathrm{ac}$ in size and before 10 o'clock the next morning [1]. This long tradition of fire suppression, together with even-aged forest structures left from intensive logging, significantly altered fuel loadings and vegetation composition in many fire-dependent ecosystems across the western USA. Currently, large amounts of accumulated fuels contribute to conditions for the recent uncontrollable catastrophic wildfires that threaten communities, wildlife, and natural resources. Since the year 2000, almost three million hectares have burned every year in the USA, more than double the average annual areas burned during the previous three decades [2].

Fuel treatments such as commercial timber harvest, mechanical thinning, mastication, and prescribed burning are recognized as practices that reduce hazardous fuels and achieve restoration objectives. The Healthy Forests Restoration Act of 2003 enacted in the USA provided extensive opportunities for hazardous fuel reduction projects to reduce the risk of catastrophic wildfires [3]. The US Forest Service (USFS) and Department of the Interior (DOI) have treated 21.2 million hectares of federal lands between 2001 and 2013, approximately 1.6 million hectares a year [4]. Wildfire management funding in 2014 accounts for $56 \%$ of the USFS's entire budget and approximately $8 \%$ of the DOI budget [5]. Despite efforts of fuel reduction and forest restoration treatments, USFS estimated that up to $42 \%$ of the entire national forests (approximately 33 million hectares) still need restoration 
treatments [6]. This need for fuel treatments is unlikely to decline due to vegetation regrowth and climate change impacts such as extended dry seasons.

As wildfire concerns grow and the need for understanding fire and fire management effects are widely recognized, large research efforts have been undertaken and significant progress has been made in wildfire behavior modeling and fire effect tools. USFS Fire Sciences Laboratory located in Missoula, MT, continues to develop cutting-edge fire models, while the interagency Joint Fire Science Program (JFSP) administered by DOI and USFS has been an effective science funding and information dissemination program. JFSP supports emerging wildland fire research and distributes research findings to help land managers develop effective fire management and fuel treatment activities.

Due to the complexity of fuel treatment planning, however, most fire models and tools in use today were designed to address limited aspects of fuel management. Integrated fuel treatment planning and optimization methods have yet to be extensively developed and used. For example, BehavePlus [7], NEXUS [8], FIREHARM [9], FARSITE [10], FlamMap [11], and many other computer simulation models were developed to compute fire behavior and spread at a treatment unit level or across a landscape.

These models do not consider temporal changes of fuels and vegetation. Missing the vegetation changes over time such as growth, mortality, and stand structure leaves longterm effects of fuel treatments unaddressed. In contrast, the Fire and Fuels Extension (FFE) to the Forest Vegetation Simulator (FVS) [12] is able to predict stand-level vegetative growth over time, but does not model fire movement between stands. Missing the spatial distribution of stands prevents the evaluation of treatment effects on a landscape level. BlueSky [13] modeling package computes fire emissions and smoke dispersion to assess smoke impacts from prescribed burns and wildfires, but does not evaluate spatial and temporal effects of fuel treatments on mitigating wildfire risk.

Land managers who need to make decisions about what, where, when, and how to perform fuel treatments to mitigate current and future fire risk must run multiple models in order to analyze and develop effective fuel treatment plans. Most of these tools are highly sophisticated, demand massive data input, and require a significant amount of time to learn and maintain. As a result, fuel treatment planning is often limited to the knowledge and experience of the planning team without any extensive search for the most effective fuel treatment operations.

Forest fuels treatment planning would likely benefit from use of an operations research approach such as optimization theory and applications. Very few studies, however, apply optimizations to fuel treatment planning mainly because of the extreme complexity of such planning problems. This paper describes the problem complexity in terms of essential considerations when deciding where, when, and how to perform fuel treatments. Previous studies published in mainstream peer-reviewed journals are summarized, identified by their unique contributions, assumptions, and simplifications. This paper focuses on past attempts to optimize the placement, timing, and methods of fuel treatments for the purpose of reducing wildfire risk potential across a landscape. Other fire-related planning efforts, such as planning of fire suppression activities or forest management planning models partly incorporating wildfires, were already summarized in two recent review papers $[14,15]$. The author promotes future research and development that fill the current gaps and incorporate both temporal and spatial effects, as well as operational constraints in forest fuel treatment analysis and planning.

\section{Complexity of Fuel Treatment Planning}

Considering spatial and temporal effects of forest fuel treatments to optimize landscape-level planning is a daunting task. Figure 1 summarizes decision variables, fuel treatment effects, and practical considerations that were taken into account in the recently developed fuel treatment optimization models.

The effects of fuel treatments on reducing fire risk change dynamically with many internal and external variables that often involve uncertainties. For example, treatment effects within a single forest unit vary with pre-treatment condition, treatment method, and post-treatment condition of the unit $[16,17]$. A single fuel treatment also influences fire behavior in its neighboring areas. A series of treatments affect fire growth potential across a landscape; overall effects may vary depending on treatment location relative to other treatment areas, untreated areas, topography, and many other locational factors [18].

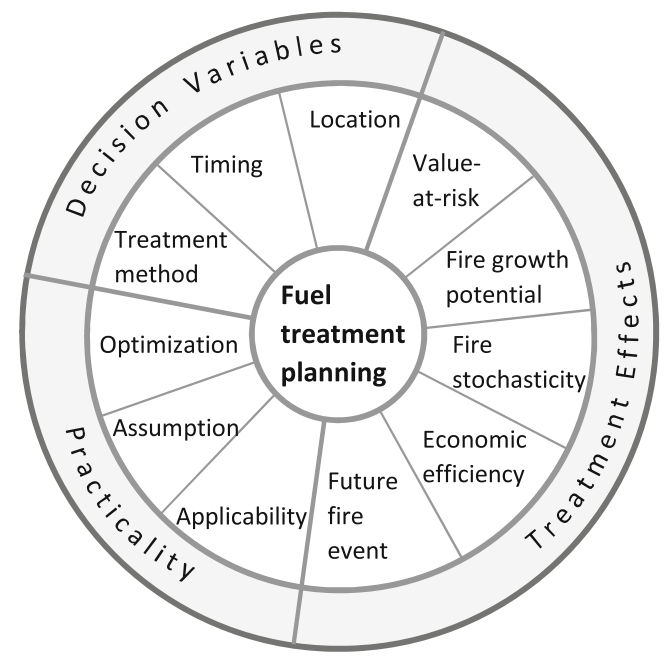

Fig. 1 Examples of decision variables, fuel treatment effects, and practicality issues that are typically considered in the past fuel treatment planning and optimization model development 
Timing of treatment adds another dimension of complexity in fuel treatment planning and optimization. Past treatments influence current fuel treatment decisions designed for future effects. Fuels and vegetation across a landscape change over time, with or without treatments. Treatment effects within the unit and on neighboring areas may diminish as time elapses since vegetation grows back [19].

Treatment method is an important decision variable when multiple methods are possible for a treatment unit. Prescribed burning can be an effective tool to reduce understory vegetation and surface fuels in many forests, although a very narrow burning window and smoke management are often challenges associated with this treatment technique. Mechanical thinning or timber harvesting activities have been used to reduce canopy fuels and can be an effective fuel management tool when used with understory burning [17]. Mechanical mastication of fuels and prescribed burning is recognized as a cost-efficient and effective method to alter fuel loadings [20].

Fire stochasticity related to likelihood of fire ignition and different weather events makes it difficult to integrate stochastic fire modeling results into a spatial optimization framework because a large number of fire simulations are often required to effectively address such uncertainties [21]. Future fire event is a stochastic disturbance that can significantly change vegetation and forest production potential across the landscape; a major fire event can affect future vegetation management decisions, especially in long-term planning [22•]. Due to the random nature of wildfires, it is difficult to predict future fires in spatial and temporal contexts.

Value at risk assigned to each land parcel is often used to estimate potential loss values due to fire risk or protection values by fuel treatments $[23,24]$. These values can provide alternative measures of success in fuel treatments, but proper risk assessment is crucial to reasonable quantification of loss or protection values. Cost and benefit analysis of fuel treatments, integrated with stochastic fire modeling, has been used to address long-term economic efficiency of the current fuel treatment decisions [22•]. Cost-efficiency of fuel treatments is an important decision criterion and key factor determining project feasibility.

Finding the optimal treatment layout across a landscape, over multiple time periods, is not a simple task. Optimization or selection of one treatment plan among many possible alternatives is methodologically and practically challenging, even when many assumptions are made to simplify the problem. Some studies attempted to use mathematical programming approaches, such as mixed integer programming and dynamic programming [22•, 24]; others used heuristic optimization techniques for solving large combinatorial problems [25, $26 \bullet \cdot]$. Some past studies developed optimization methods only based on hypothetical settings, not real-world applications. Applicability of tools and models is certainly essential to land management agencies and other potential users.

\section{Recent Fuel Treatment Optimization Modeling Efforts}

Mark Finney with USFS Fire Sciences Laboratory pioneered fuel treatment planning. In his early work, Finney proved the efficiency of strategically placed treatments in disrupting fire growth across a landscape, simulating fire spread on simple hypothetical landscapes with fuel treatments in various geometric arrangements [18]. He later developed an optimization algorithm to identify treatment locations across a landscape that most efficiently disrupts fire growth while meeting a given treated portion of total landscape area [27].

The optimization algorithm starts with dividing the landscape into a series of parallel strips, then computes fire growth and minimum travel routes within each strip. Areas with the greatest impact on blocking fire paths in the strip are prioritized as treatment units. Although the algorithm was able to identify efficient fuel treatment locations, model queries are limited to one strip at a time due to the computational challenge; overall treatment effects across the entire landscape were not examined. In addition, no temporal aspects of treatments were considered. The shape of each selected treatment area was theoretically developed based on fire paths, not by terrain or operational unit boundaries, limiting the applicability of the algorithm in actual land management settings.

Palma et al. [23] developed a method to evaluate relative effects of timber-harvesting treatment in each individual forest stand, ranking treatments by their effectiveness of protecting values, expressed as reductions in loss values across a landscape. Their method did not optimize fuel treatments, but ranked forest stands by their potential contribution to slowing down fire spread. Potential contribution of a harvesting block was derived from changes in fire risk in the areas affected by the block. Fire risk was calculated as a function of fire occurrence probability, the values at risk, and reduced fire spread probabilities by the treatment block.

Palma's study addresses the effects of a single-harvesting block on the entire landscape by identifying all the areas that the treatment block could potentially affect. The method computes shortest fire paths between all possible pairs of grid cells on the landscape and identifies areas where shortest paths go through the subject treatment block. The potential savings on expected loss by one single block are then calculated based on the comparison between the untreated and treated harvesting block cases.

One weakness of the method is that it assumes only one harvesting block is treated at a time, excluding potential synergetic effects from multiple harvesting blocks. In practice, more than one harvesting block is treated, and overall treatment effects across the landscape would be different depending on combinations of harvesting blocks and the sequence of their treatments. The ranks of individual harvesting blocks as a single treatment might not serve as a valid measure when multiple blocks are to be treated. 
Acuna et al. [28•] incorporated this ranking method into a forest management planning model. The model is an iterative forest fire management planning system that models fire ignition, suppression and spread, ranks harvesting units, and spatially schedules harvest activities to maximize economic returns. Using the mixed integer linear programming approach, the model optimizes treatment locations and timing based on pre-computed distribution of burn probability and ranking of harvesting blocks. The model applicability carries the same caveat as Palma et al. [23] and does not address fire dynamics affected by multiple treatment locations over multiple time periods.

Wei et al. [24] used a probabilistic approach to estimate expected loss value of each raster grid cell across a landscape. Their study simplifies fire spread between cells. They assume a cell can burn by either a fire ignited in the cell or entered only from three adjacent cells located in the wind direction. This simplification allows the modelers to estimate fire risk in each cell based on the probability of fire occurrence and conditional probability of fire spread from adjacent cells as linear functions. The fuel treatment placement problem becomes a mixed integer programming problem with an objective function to minimize the total expected fire loss across the landscape. Distribution of values at risk, fire ignition risk, fire intensity, and the conditional probability for fire entering from adjacent cells were all driving factors for the objective function. Limited treatment budget was considered as a constraint.

Their model was the first trial to formulate the fuel treatment planning problems using a mathematical programming approach. However, their optimization model does not schedule treatments, and the grid cell size used in the study (i.e., $23 \mathrm{ha}$ ) is probably too large to be practical; it may not capture variability in topography and vegetation that can affect wind direction, fuel loading, and resulting fire spread.

Kim et al. [25] used a heuristic optimization technique called Great Deluge [29] to schedule timber management activities in specific spatial patterns across a landscape. They evaluated subsequent and cumulative effects of scheduled management activities on changing wildfire behavior using multiple fire simulations. They attempted to address wildfire risk mitigation within the context of spatially explicit forest management planning, where desirable timber harvest volumes are pursued in each planning period for a given landscape. Their selection of timber harvest units was based on both even flow of desirable harvest volume of timber and spatial patterns of harvest units.

The study of Kim et al. concluded that forest management activities designed to meet timber management goals were not effective enough to mitigate landscape-scale wildfire behavior despite the spatial arrangement of harvesting units. One of the lessons learned from their study is that spatial arrangement of treatments could be a necessary prerequisite, but not sufficient condition for efficient landscape-level fire mitigation. Unlike a homogeneous, hypothetical landscape, spatial arrangement of fuel treatments that simply follow a geometric pattern may not be effective in fire mitigation on a heterogeneous landscape. The specificity of individual treatments in terms of location, timing, topography, and conditions of neighboring areas needs to be considered as a whole, along with its spatial pattern to realize greater fire risk mitigation benefits.

Konoshima et al. [22•] applied a spatial dynamic optimization model to a hypothetical landscape consisting of seven equal-sized hexagonal management units, exploring optimal patterns of fuel treatment under various physical and economic conditions. One of the unique contributions of their study is that it incorporates stochastic future fire events that are influenced by fuel treatment decisions. In their optimization framework, treatment locations are determined for each of two 10year periods by maximizing both net revenue in the current period (i.e., timber harvest incomes minus treatment costs) and the expected net present value of future timber production. Incorporation of future fire events into a decision model allows the planners to develop different trajectories that might occur from stochastic events, probabilistically analyzing future benefits and costs of the current fuel management activities.

The study suggests it could be more economically beneficial to shift fire management focus from protection against fire spread to protection of on-site resource value as the fire environment becomes more severe or as net value of the management unit increases. Similar to other studies, the study simplified the planning problem to reduce complexity. The simplifications include seven hypothetical management units of equal size, fuel treatments available only in the first planning period, and no future fire events considered beyond the 20 year planning horizon.

Finney et al. [30] integrated the existing vegetation, fire, and optimization models into one system to schedule fuel treatments while addressing spatial and temporal effects of treatments. Finney's treatment optimization algorithm [27] was used to place fuel treatments where the treatment efficiently disrupts major fire paths across the landscape for each single planning period. The FVS was used to simulate changes in vegetation and fuels based on the selected fuel treatment locations from the previous planning period. Using these two models, sequentially and iteratively, the system develops treatment decisions for one planning period at a time, evaluates the landscape effects of the selected treatments, and then grows the landscape to the next period, providing vegetation and fuel data for fuel treatment decisions of that period. Their system is the first attempt to fully address both spatial and temporal fire dynamics affected by fuel treatments over multiple time periods.

Although this system is able to schedule fuel treatments across a given landscape, the forward decision approach makes earlier period decisions dominate the decisions to be 
made for subsequent periods. Limitations in practical considerations for field implementation, i.e., treatment unit boundaries, ownerships, and relative values of lands, also reduce the applicability of this model.

González-Olabarria and Pukkala [31••] developed an iterative optimization approach that simulates random fires to estimate burn probability of each hexagon in the landscape, then uses these probabilities in optimizing fuel treatments over multiple time periods. They developed a fire spread simulator using cellular automation modeling that spreads fire from a random ignition point to its adjacent hexagons with a probability based on a function of the slope and the characteristics of the neighboring hexagon. An initial fire spread simulation is performed for the entire planning horizon prior to treatment scheduling. The simulation is repeated after an optimal harvest schedule is developed to reflect the effects of selected fuel treatments on burn probability across the landscape. This iterative process continues until burn probability and treatment schedule have stabilized.

Their approach uses a unique fire damage model to estimate fire-related damage in forest stands. Burn probability in each hexagon together with the fire damage enables the user to estimate the expected income from selected timber harvest operation schedules in each hexagon while considering timber values, harvesting costs and potential fire damage. The net income was used as a measure of success as it reflects economic efficiency of treatments as well as increased fire resistance due to selected treatments. A simulated annealing algorithm [32] was employed to optimize harvest treatments and meet a given target harvest volume in each period.

The approach optimizes spatial and temporal placement of harvest treatments while addressing fire stochasticity, fire dynamics across the landscape, and economic efficiency of treatments. Similar to Acuna et al. [28•], timber management was the main objective of this approach and other fuel treatment methods, such as prescribed burning, were not considered. Another limitation may be the simplified fire spread simulation employed in the approach. The simulation appears to consider only ground slope for quick calculation of the rate of fire spread, excluding weather, aspects, and other potential factors that may affect fire growth and dynamics.

Chung et al. [26••] developed a decision support system called OptFuels for fuel treatment planning. Similar to Finney et al. [30], the system uses the FVS and its Fire and Fuels Extension (FFE-FVS) to project forest stands into the future, with and without fuel treatments, and compute the fuel parameters needed for fire growth modeling. Instead of growing the landscape in each planning period based on the treatments selected for the previous period (i.e., forward approach), OptFuels develops all possible treatment schedules for individual units based on user-defined rules and time periods prior to optimization.
For example, if a treatment unit is eligible for mechanical thinning followed by prescribed burning in any of two planning decades, all possible combinations of treatment options and timing for the unit would be the following: "no action" throughout the planning horizon, "thinning and prescribed burning" in the first decade, and "thinning and prescribed burning" in the second decade. If more treatment methods $(n)$ and time periods $(t)$ are available for a single unit, the total number of possible treatment schedules for the unit would be $(n \times t)+1$. This assumes that the unit can receive only one treatment throughout the planning horizon or no action at all.

Before the optimization process begins, the system runs FFE-FVS on each of the possible treatment options for all the eligible units in a given landscape and develops a database with the simulation results for use during optimization. OptFuels uses a simulated annealing heuristic technique [32] to solve the large combinatorial problem. The objective function is to minimize the total expected loss across the landscape over multiple time periods. The landscape is rasterized into grid cells, and expected loss value for each grid cell is computed as a function of a user-defined value at risk. Percentage loss varies with fire intensity and probability that fire duration is long enough for a modeled fire to reach the grid cell. The total expected loss is then summed across the grid cells representing the landscape.

The Dynamic Link Library (DLL) version of FlamMap is used for fire growth modeling in order for OptFuels to seamlessly run FlamMap in real time for solution evaluation. FlamMap contains an option called minimum travel time, which is a fire growth simulator that uses the shortest path algorithm to compute how fast one fire or a band of multiple fires move across a landscape. As the outcomes of the FlamMap simulation, fire arrival time and fire intensity per grid cell are transferred to OptFuels for the calculation of total expected loss across the landscape for a given treatment plan.

OptFuels begins with a no-action scenario across the landscape, providing an initial no-action solution for comparison. Then, the system randomly selects treatment units and assigns one of the eligible treatment schedules pre-developed for each unit until the total treatment acres in each planning period exceeds the user-defined minimum treatment acres. This step generates a random feasible solution that is used as an initial solution in the simulated annealing algorithm. The algorithm involves a large number of iterations. In each iteration, the current solution is slightly modified by replacing one or more treatment locations or treatment schedule options to form a new solution for evaluation. This solution process is repeated until a user-defined ending criterion is met.

Although the heuristic algorithm does not guarantee the optimality of the final solution, it improves the solution quality throughout the search process (Fig. 2). In addition, 
Fig. 2 An example showing the performance of OptFuels on solution improvement. A total of 3147 alternative solutions were created and evaluated throughout the optimization process in this example. At the end of the process, the quality of the best solution representing total expected loss value was improved by $62 \%$ from no-action solution and $48 \%$ from the random initial solution that had the similar amount of areas treated as the final best solution (adapted from Chung et al. [26••])
Improvement in Solution Quality

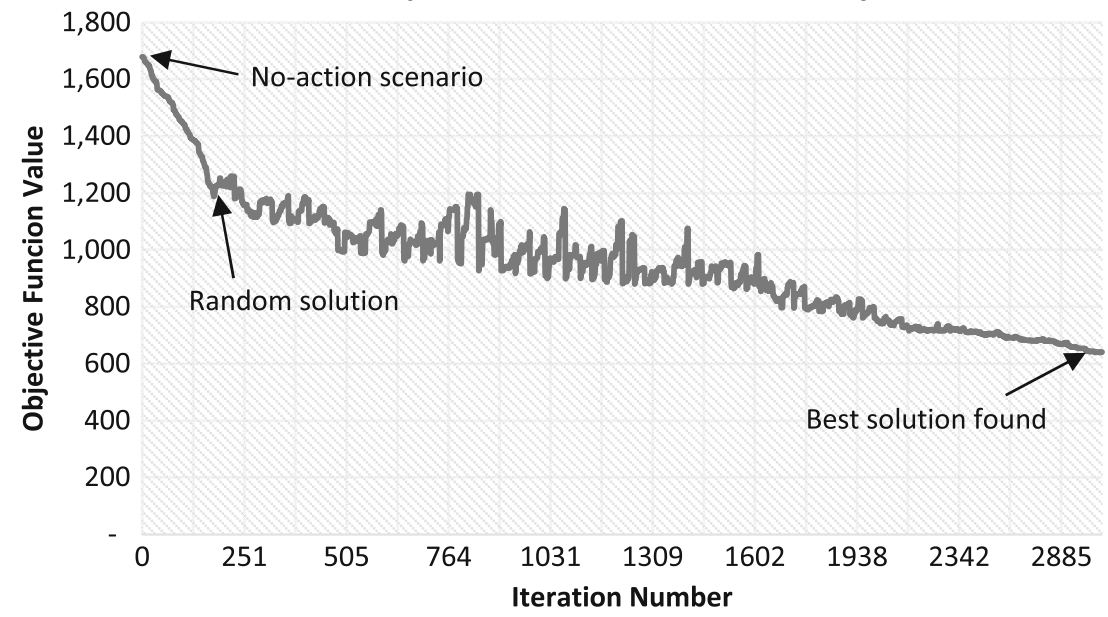

the ability to modify treatment schedules during the process enables the algorithm to overcome the forward-only decision approach in multiple time period planning. The system takes the deterministic approach in fire growth modeling by using pre-defined fire ignition locations and weather scenarios. The model does not consider fire stochasticity, nor impacts of future fire events on the landscape.

\section{Discussion}

Although many studies in the past attempted to incorporate wildfires into forest management planning models, most of them considered wildfires as constraints or used fire growth models to assess model outcomes [15]. Only a handful studies actually integrated fire spread logics or fire behavior models into the fuel treatment optimization framework with various

Table 1 Important considerations in fuel treatment optimization addressed in each of the previous studies

\begin{tabular}{|c|c|c|c|c|c|c|c|}
\hline Considerations & $\begin{array}{l}\text { Finney et al. } \\
{[30]}\end{array}$ & $\begin{array}{l}\text { Wei et al. } \\
{[24]}\end{array}$ & $\begin{array}{l}\text { Kim et al. } \\
{[25]}\end{array}$ & $\begin{array}{l}\text { Acuna et al. } \\
{[28 \cdot]}\end{array}$ & $\begin{array}{l}\text { Konoshima et al. } \\
{[22 \bullet]}\end{array}$ & $\begin{array}{l}\text { González- } \\
\text { Olabarria } \\
\text { and Pukkala } \\
{[31 \bullet \bullet]}\end{array}$ & $\begin{array}{l}\text { Chung et al. } \\
{[26 \bullet \bullet]}\end{array}$ \\
\hline $\begin{array}{l}\text { Fuel treatment } \\
\text { location }\end{array}$ & $\checkmark$ & $\checkmark$ & $\checkmark$ & $\checkmark$ & $\checkmark$ & $\checkmark$ & $\checkmark$ \\
\hline $\begin{array}{l}\text { Timing of fuel } \\
\text { treatment }\end{array}$ & $\checkmark$ & & $\checkmark$ & $\checkmark$ & $\checkmark$ & $\checkmark$ & $\checkmark$ \\
\hline $\begin{array}{l}\text { Fuel treatment } \\
\text { method }\end{array}$ & $\checkmark$ & & $\checkmark$ & & $\checkmark$ & & $\checkmark$ \\
\hline Optimization & Heuristic & Mixed integer & Heuristic & $\begin{array}{l}\text { Iterative mixed- } \\
\text { integer }\end{array}$ & Dynamic & Heuristic & Heuristic \\
\hline Objective function & $\begin{array}{l}\text { Min. fire } \\
\text { spread rate }\end{array}$ & $\begin{array}{l}\text { Min. expected } \\
\text { loss }\end{array}$ & $\begin{array}{l}\text { Min. deviation } \\
\text { from timber } \\
\text { target and } \\
\text { treatment } \\
\text { patterns }\end{array}$ & Max. net revenue & $\begin{array}{l}\text { Max. net present } \\
\text { value }\end{array}$ & $\begin{array}{l}\text { Max. expected } \\
\text { net revenue }\end{array}$ & $\begin{array}{l}\text { Min. expected } \\
\text { loss }\end{array}$ \\
\hline $\begin{array}{l}\text { Fire dynamics and } \\
\text { growth }\end{array}$ & $\checkmark$ & & & & & $\checkmark$ & $\checkmark$ \\
\hline Value at risk & & $\checkmark$ & & $\checkmark$ & $\checkmark$ & & $\checkmark$ \\
\hline Fire stochasticity & $\checkmark$ & $\checkmark$ & & $\checkmark$ & $\checkmark$ & $\checkmark$ & \\
\hline $\begin{array}{l}\text { Long-term } \\
\text { economic } \\
\text { efficiency }\end{array}$ & & & & & $\checkmark$ & & \\
\hline Future fire events & & & & & $\checkmark$ & & \\
\hline $\begin{array}{l}\text { Applicability } \\
\text { potential }\end{array}$ & Medium & Medium & High & Medium & Low & Medium & High \\
\hline
\end{tabular}


levels of detail or different issues of concern. Some studies simplified the problem by looking at only placement of fuel treatments, while other studies addressed treatment scheduling over multiple time periods at the expense of simplifying fire spread calculation across the landscape.

Most of the past fuel treatment optimization work were developed in the USA. This reflects the nation's policy changes in federal wildland fire management over the last two decades, making hazardous fuel reduction and forest restoration a dominant management objective in many dry, firedependent ecosystems in the western USA.

Table 1 organizes the previous studies reviewed in this paper into a matrix aimed at identifying fuel treatment planning issues addressed in each study based on the authors' subjective interpretation. Most of them attempted to address both spatial and temporal arrangements of fuel treatments. The treatment methods considered by Wei et al. [24], Acuna et al. [28•], and González-Olabarria and Pukkala [31••] were focused around timber management rather than fuel treatment, whereas Finney et al. [30] and Chung et al. [26••] mainly dealt with fuel reduction and restoration treatments.

Mathematical programming approaches were used only when fuel treatment effects on fire dynamics were simplified in a linear model or translated into burn probability computed prior to optimization $[24,28 \cdot]$ or when the landscape is simple enough to track down possible combinations of activities [22•]. Heuristic approaches were used to deal with non-linear effects of fuel treatments or large combinatorial problems when spatial and temporal dynamic changes of fire growth potential were considered across a complex landscape. Fire protection value or value at risk was often used as a measure of success that guides fuel treatment decisions. Some studies employed a stochastic approach to estimate expected loss values by computing the distribution of fire ignition and occurrence probabilities based on a large number of fire growth simulations under varying weather conditions $[22 \bullet, 24,28 \bullet, 30,31 \bullet \bullet]$, while others used a deterministic approach to avoid large fire simulations [25, 26••].

The long-term economic efficiency of fuel treatments appears difficult to address because it requires modeling future disturbance as a function of current fuel treatments. So far, only one study attempted to address the issue by modeling possible trajectories of future fires across the landscape [22•].

Most of the existing studies introduced optimization approaches as proof of concept with limited applications. The work of Chung et al. [26••] is one of the very few studies that has been incorporated into a decision support system currently available for use in real treatment planning efforts [33]. As Martell [14] pointed out, collaboration with land and fire managers on development of decision support tools would be essential to address the practicality of developing methods for use by operations and land managers.

It is hoped that future studies will build on these previous efforts and develop more efficient and integrated optimization methods that can address multiple concerns simultaneously while producing effective fuel treatment plans for field implementation.

\section{Conclusions}

Optimizing forest fuel treatments to reduce wildfire risk over long periods and across a large landscape is a daunting, yet necessary task. The unpredictable nature of fire starts, the complex factors directing fire behavior, and the fuel treatment effectiveness are difficult to measure. Computer models developed during the last decade to optimize forest fuel treatments address only limited concerns. Most existing optimization models still suffer from problem complexity and a computationally intensive process of fire modeling, making them almost impractical for field applications.

Building on these past efforts, future research must result in more comprehensive, field-ready forest fuel treatment projects. More reliable and field-verified data will result in more efficient fire models, as fire managers, researchers, and practitioners gain experience with wildfire behavior and the effects of fuel-treated acres. Technical improvements in computer hardware and software in tandem with scientific advances in optimization techniques can reduce computational expenses for solving challenging optimization problems.

Large forest landowners, primarily public agencies using landscape-level planning, will benefit most from these advances in fuel treatment optimization. Integrated optimization modeling of fuel treatments to mitigate the risk of wildfire will be a key research area for fire-dependent ecosystems. Continued monitoring and research will be necessary to demonstrate that land managers can trust and rely on the output of computer analysis as results match what is observed in the field.

\section{Compliance with Ethics Guidelines}

Conflict of Interest Dr. Chung states that he has no conflicts of interests to declare.

Human and Animal Rights and Informed Consent This article contains no studies with human or animal subjects performed by the author.

\section{References}

Papers of particular interest, published recently, have been highlighted as:

- Of importance

•• Of great importance

1. U.S. Dept. of the Interior, Office of Policy Analysis. 2012. Wildland fire management program benefit-cost analysis: a review of relevant literature. 70p. http://www.doi.gov/ppa/upload/Wildland fire literature_review_060812FINAL.pdf. 
2. U.S. Forest Service. 2013. Fiscal year 2014 budget overview. http:// www.fs.fed.us/aboutus/budget/2014/FY2014ForestServiceBudget Overview041613.pdf.

3. Healthy Forest Restoration Act of 2003. 2003. Pub. L. 108-148, 117 Stat. 1887

4. Forests and Rangelands. 2014. Hazardous fuels reduction and landscape restoration accomplishments fiscal years (FY) 2001-2013. 1p. http://forestsandrangelands.gov/resources/reports/documents/ 2013/HFR_DOI_FS_Accomplishments2001-2013.pdf.

5. Bracmort, K. 2014. Wildfire protection in the wildland-urban interface. Congressional research service report RS21880. 10p.

6. U.S. Forest Service. 2012. Increasing the pace of restoration and job creation on our national forests. U.S. Dept. of Agriculture. 8p. http://www.fs.fed.us/publications/restoration/restoration.pdf.

7. Andrews PL. Current status and future needs of the BehavePlus fire modeling system. Int J Wildland Fire. 2014;23(1):21-33.

8. Scott JH. NEXUS: a system for assessing crown fire hazard. Fire Manag Notes. 1999;56:20-4.

9. Keane RE, Drury SA, Karau EC, Hessburg PF, Reynolds KM. A method for mapping fire hazard and risk across multiple scales and its application in fire management. Ecol Model. 2010;221:2-18.

10. Finney MA. 2004. FARSITE: fire area simulator - model development and evaluation. U.S. Dept. of Agriculture, Forest Service, Rocky Mountain Research Station, Research Paper RMRS-RP-4 Revised. Ogden, UT.

11. Finney MA. An overview of FlamMap modeling capabilities. In: Andrews PL, Butler BW, editors. Proceedings of conference on fuels management - how to measure success. Rocky mountain research station proceedings RMRS-P41. Ogden: U.S. Dept. of Agriculture, Forest Service, Rocky Mountain Research Station; 2006. p. 213-20.

12. Reinhardt ED, Crookston NL. The fire and fuels extension to the forest vegetation simulator. U.S. Dept. of Agriculture, Forest Service, Rocky Mountain Research Station, General Technical Report RMRS-GTR-116. Ogden, UT; 2003.

13. O’Neill SM, Ferguson SA, Wilson R. The BlueSky smoke modeling framework. Orlando: American Meteorological Society, 5th Symposium on Fire and Forest Meteorology; 2003.

14. Martell D. The development and implementation of forest fire management decision support systems in Ontario, Canada: personal reflections on past practices and emerging challenges. Math Comput For Nat Res Sci. 2011;3(1):18-26.

15. Bettinger P. An overview of methods for incorporating wildfires into forest planning models. Math Comput For Nat Res Sci. 2010;2(1):4352.

16. Agee JK, Skinner CN. Basic principles of forest fuel reduction treatments. For Ecol Manag. 2005;211:83-96.

17. Prichard SJ, Peterson DL, Jacobson K. Fuel treatments reduce the severity of wildfire effects in dry mixed conifer forest, Washington. USA Can J For Res. 2010;40:1615-26.

18. Finney MA. Design of regular landscape fuel treatment patterns for modifying fire growth and behavior. For Sci. 2001;47(2):219-28.

19. Ager AA, McMahan AJ, Barrett JJ, McHugh CW. A simulation study of thinning and fuel treatments on a wildland-urban interface in Eastern Oregon. USA Landsc Urban Plan. 2007;80:292-300.
20. Clark R. 2009. Masticating fuels: effects on prescribed fire behavior and subsequent vegetation effects. JFSP Briefs. Paper 62. http:// digitalcommons.unl.edu/jfspbriefs/62/.

21. Ager AA, Vaillant NM, Finney MA. A comparison of landscape fuel treatment strategies to mitigate wildland fire risk in the urban interface and preserve old forest structure. For Ecol Manag. 2010;259:1556-70.

22. Konoshima M, Albers HJ, Montgomery CA, Arthur JL. Optimal spatial patterns of fuel management and timber harvest with fire risk. Can J For Res. 2010;40:95-108. This study attempted to address the long-term economic efficiency of fuel treatments by incorporating future fire events into fuel treatment optimization.

23. Palma CD, Cui W, Martell DL, Robak D, Weintraub A. Assessing the impact of stand-level harvests on the flammability of forest landscapes. Int J Wildland Fire. 2007;16:584-92.

24. Wei Y, Rideout D, Kirsch A. An optimization model for locating fuel treatments across a landscape to reduce expected fire losses. Can J For Res. 2008;38:868-77.

25. Kim Y-H, Bettinger P, Finney MA. Spatial optimization of the pattern of fuel management activities and subsequent effects on simulated wildfires. Eur J Oper Res. 2009;197:253-65.

26.• Chung W, Jones G, Krueger K, Bramel J, Contreras M. Optimizing fuel treatments over time and space. Int J Wildland Fire. 2013;22: 1118-33. This study incorporated the well-adapted fire and vegetation growth models into a fuel treatment optimization system to realistically assess spatial and temporal effects of fuel treatments. The modeling concept provided the basis for the currently available decision support system for fuel treatment planning.

27. Finney MA. A computational method for optimising fuel treatment locations. Int J Wildland Fire. 2007;16:702-11.

28. Acuna MA, Palma CD, Cui W, Martell DL, Weintraub A. Integrated spatial fire and forest management planning. Can J For Res. 2010;40:2370-83. This study incorporated fire stochasticity into an iterative fuel treatment optimization process where the potential effects of fuel treatments across the entire landscape are considered in prioritizing harvest blocks.

29. Dueck G. New optimization heuristics: the great deluge algorithm and the record-to-record travel. J Comput Phys. 1993;104:86-92.

30. Finney MA, Seli RC, McHugh CW, Ager AA, Bahro B, Agee JK. Simulation of long-term landscape-level fuel treatment effects on large wildfires. Int J Wildland Fire. 2007;16:712-27.

31.• González-Olabarria J-R, Pukkala T. Integrating fire risk considerations in landscape-level forest planning. For Ecol Manag. 2011;261:278-87. This study attempted to fully integrate timber and fuels management by incorporating timber incomes, treatment costs, and fire damage potential into a single objective function while simulating fire spread and adjusting fire probability during the optimization process.

32. Kirkpatrick S, Gelatt CD, Vecchi MP. Optimization by simulated annealing. Science. 1983;220:671-80.

33. Shari A. 2011. Optimizing the location of fuel treatments over time at landscape scales. JFSP Briefs. Paper 131. http://digitalcommons. unl.edu/jfspbriefs/131. 